\title{
X. Die Vergeltung
}

Die Beschäftigung mit der Frage der Bestrafung der NS-Verbrecher setzte unmittelbar nach Kriegsende im Mai 1945 ein und in gewissen Fällen schon vorher: So trug etwa die Beteiligung jüdischer Einheiten an den Kämpfen gegen das nationalsozialistische Deutschland zweifellos bereits Züge von Vergeltung und (Selbst-) Justiz. Im selben Geist gingen nach dem Krieg selbsternannte „Rächer" auf eigene Faust und ohne jede rechtliche Grundlage und Rechtfertigung mit gewalttätigen Aktionen gegen Nationalsozialisten und deren Kollaborateure vor. Erst die Gründung des Staates Israel setzte diesen Aktivitäten ein Ende.

\section{Grundlagen der Vergeltungsjustiz in Israel}

Vergeltungsgesetze und Kriegsverbrecherprozesse waren verbreitete Erscheinungen nach dem Krieg, die Nürnberger Prozesse 1945/46 nur ein Beispiel davon, wenn auch das wichtigste. Eines der Hauptthemen in Nürnberg, aber nicht nur dort, war der Völkermord an den Juden. In ganz Osteuropa wurden Todesurteile gegen Verantwortliche für die Deportation und Vernichtung der Juden gefällt. Doch an keinem dieser Prozesse beteiligten sich Juden als "Vertreter des Judentums". Der vermutlich erste Prozeß von Juden gegen einen jüdischen NS-Kollaborateur fand Ende 1945 im DP-Lager Landsberg, Bayern statt. ${ }^{1} \mathrm{Zu}$ ähnlichen Retributionsprozessen gegen Kollaborateure kam es später auch in Israel. In etwa zweihundert Fällen wurden Neueinwanderer von Mitbürgern als „Kollaborateure" erkannt, angezeigt, vor Gericht gestellt und zu Gefängnisstrafen verurteilt. Doch schon nach kurzer Zeit wurden die bestehenden Gesetze des jungen Staates als unzulänglich im Umgang mit dieser ganz besonderen Art von Verbrechen eingestuft.

Der sich der Vergeltung und der Holocaust-Erinnerung widmende nationalreligiöse Abgeordnete Rabbiner Mordechai Nurock legte in der ersten Knesset im Oktober 1949 einen Gesetzesvorschlag für die Verfolgung von Kriegsverbrechern vor. ${ }^{2}$ Anfang Dezember 1949 diskutierte und verabschiedete das israelische Kabinett die UNO-Konvention gegen Völkermord und legte sie anschließend ebenfalls der Knesset vor. ${ }^{3}$ Am 26. Dezember brachte der Justizminister die Vorlage zur ersten Lesung ein, und kurz darauf folgte ein Gesetzesvorschlag für die Verfolgung von Nationalsozialisten und deren Helfer. ${ }^{4}$

Das am 1. August 1950 einstimmig verabschiedete Gesetz ermöglicht als einziges israelisches Zivilgesetz die Verhängung der Todesstrafe. Zudem ignoriert es

1 Digest of Jewish Camp Papers vom 16. 1. 1946, AJA, WJC, U-245.

2 KNESSET-ProtoKolle [Original hebr.], 1. Knesset, 90. Sitzung vom 29. 10. 1949.

3 Protokoll der Kabinettssitzung Nr. 12/310 vom 5. 12. 1949, ISA, 7263/4.

4 KNESSET-PROTOKOLle [Original hebr.], 1. Knesset, 131. Sitzung vom 27. 3.1950. 
das juristische Grundprinzip „Nulla poena sine lege“ (keine Strafe ohne Gesetz), d. h. es gilt auch für Verbrechen, die vor Inkrafttreten des Gesetzes und außerhalb Israels begangen wurden. Mit anderen Worten, das Gesetz sieht Strafen für Handlungen vor, die zur Tatzeit, formal betrachtet, legal waren, bzw. Strafen für Taten, die außerhalb der Grenzen des Staates Israel und vor dessen Gründung begangen wurden. Begründet wird dies mit dem Sui-generis-Charakter der begangenen Verbrechen: Nie zuvor war es zu einer systematischen, ideologisch motivierten physischen Vernichtung eines ganzen Volkes gekommen, wie sie in diesem Umfang nur im Zeitalter moderner Technologie möglich ist. Diese Tat ist ohne Beispiel, „sui generis“. Entsprechend wurde eine beispiellose Strafe gefordert. Zwei Präzedenzfälle sprachen für das Gesetz: die Konvention vom 8. August 1945 "Agreement for Prosecution and Punishment of the Major War Criminals of the European Axis“, auf der die rechtliche Grundlage der Nürnberger Prozesse beruhte und die UNO-Konvention gegen den Völkermord von 1948. Dem israelischen Gesetzgeber ging es in erster Linie um die strafrechtliche Verfolgung der NS-Verbrechen gegen die Juden und anderer Judenverfolgungen im Verlaufe der Geschichte, die ungestraft geblieben waren. Es war in gewisser Hinsicht also eine jüdische Abrechnung mit der Geschichte. Das Gesetz wurde einige Male gegen NS-Kollaborateure, in den sechziger Jahren gegen Adolf Eichmann und in den neunziger Jahren gegen John Demianjuk angewandt.

\section{Israel und die Vergeltungsjustiz in der Bundesrepublik}

Die israelische Öffentlichkeit verfolgte die Strafverfahren gegen NS-Verbrecher in der Bundesrepublik Deutschland mit größter Aufmerksamkeit und zeigte sich tief beunruhigt über die aus ihrer Sicht zu nachsichtige Haltung der Besatzer und der deutschen Behörden gegenüber NS-Verbrechern. Der amerikanische Hochkommissar für Deutschland, John J. McCloy, weckte in Israel gemischte Gefühle, nachdem dieser die Entlastung, Begnadigung und Freilassung von Nationalsozialisten und Kriegsverbrechern angeordnet hatte. ${ }^{5} \mathrm{McCloy}$ war seit seiner Nominierung zum Hochkommissar dem Druck für die Begnadigung von Kriegsverbrechern ausgesetzt. Ein solcher Fall betraf den ehemaligen Staatssekretär von Ribbentrops, Ernst von Weizsäcker, dessen vorzeitige Freilassung von George F. Kennan, Bernard Gufler und Reinhold Niebuhr gefordert wurde. Der bekannte Theologe Niebuhr schrieb McCloy: „Er [Weizsäcker] wurde nur deshalb verurteilt, weil er zugegeben hatte, nach Ausbruch des Krieges nicht für die Niederlage seiner Nation gewesen zu sein. Die Art, wie Kempner die Anklage in diesen Prozessen führte, macht der amerikanischen Justiz meines Erachtens keine Ehre. Nicht die amerikanische Justiz, sondern der Groll von Flüchtlingen war hier am Werk. "6 Man kann den euphemistischen Begriff „Flüchtlinge“ hier ruhig durch „Juden“ ersetzen.

5 Drúks, John J. McCloy.

6 Niebuhr an McCloy vom 15.6. 1949, Amherst College Archives, McCloy Papers, Box $\mathrm{HC} 4$, Series 13, Folder 19. 
Jüdische und israelische Kreise fühlten sich von der zu liberalen Haltung der amerikanischen Behörden vor den Kopf gestoßen. Die Empörung erreichte ihren Höhepunkt, als sich McCloy mit Möglichkeiten der Strafmilderung für jene besonders schweren NS-Verbrecher befaßte, die in Landsberg auf die Vollstreckung ihres Todesurteils warteten oder dort eine langjährige Gefängnisstrafe absaßen. Kritiker bezeichneten diese Urteile als "Siegerjustiz" und forderten deren Umwandlung. Dies war eine Forderung, der man sich angesichts des Kalten Krieges und der Interessen der westlichen Alliierten, die ein gutes Verhältnis zur deutschen Bevölkerung unerläßlich machten, nur schwer verschließen konnte. Doch die Motive des Firmenanwalts McCloy, dem beste Verbindungen zu amerikanischen Finanzkreisen, zur amerikanischen Schwerindustrie und zur kapitalistischen Aristokratie Amerikas nachgesagt wurden, waren nicht über jeden Zweifel erhaben. Nicht vergessen war sein umstrittenes Verhältnis zu Juden und Amerikanern japanischer Abstammung im Zweiten Weltkrieg sowie sein Anteil an der Verhinderung der Bombardierung von Auschwitz, und - in anscheinend krassem Gegensatz dazu - die scharfe Verurteilung von Jakob Altmaiers Appell für die Begnadigung von Kriegsverbrechern. ${ }^{7}$ McCloys Unterstützung der Schilumim und bestimmter Entschädigungsgesetze kompensierten frühere Fehltritte lediglich bis zu einem gewissen Grad. ${ }^{8}$

Die Begnadigungen und Strafumwandlungen, die McCloy verkündete9, lösten in Israel großen Zorn im gesamten politischen Spektrum aus. Aber der Versuch der Opposition, das Thema in der Knesset zu behandeln, wurde von der Regierung verhindert. Sie befürchtete, antiwestliche linke Elemente könnten die Angelegenheit für eigene Zwecke in Zusammenhang mit dem Kalten Krieg mißbrauchen. Die Knesset begnügte sich schließlich mit einer unspektakulären Protestnote, die in klarem Kontrast zum großen Echo stand, den der oppositionelle Vorstoß in der Öffentlichkeit gefunden hatte. ${ }^{10}$ Daß McCloy bei der Umwandlung einiger Urteile aus eigener Initiative und im Rahmen seiner Vollmachten handelte, bedeutet nicht, daß seine Entscheidungen nicht im Sinne der amerikanischen Regierung waren. Eisenhower, damals Oberbefehlshaber der NATO-Streitkräfte in Europa, rief dazu auf, zwischen jenen zu unterscheiden, die wegen schrecklicher Kriegsverbrechen verurteilt worden seien, und normalen deutschen Offizieren und Soldaten, denen, wie er sich ausdrückte, „nicht das Gefühl gegeben werden sollte, daß ihre [Soldaten-]Ehre angezweifelt werde." Dazu meinte ein britischer Diplomat: „Diese Unterscheidung dürfte unumgänglich sein, wenn deutsche Sol-

7 Bericht von Dr. Gerhard Jacoby vom 12. 1. 1951, ISA, 533/7b.

8 Zu McCloy: Druks, John J. McCloy; JelineK, John McCloy; SChwartz, AtlantikBrücke; FerenCZ, Lohn des Grauens, S. 100-106; JAPANESE AMERICANS.

9 SChwarTZ, Begnadigung.

10 Edward W. Holmes an den Außenminister vom 9. 3. 1951, USNA, RG 84, Tel Aviv, Israel Consular Post, 1950-52; Edward W. Holmes und Davis, Tel Aviv, an den Außenminister vom 12. 3. 1951, USNA, 321.9, Germany; Evan an Blaustein vom 3. 3. 1951, YIVO, AJC, RG 347, GEN-10, Box 291; Evan an Dr. Goldstein vom 9. 3. 1951, CZA, Z 6/530; Evan an Richter Meier Steinbrink, den Vorsitzenden der Liga gegen Verleumdung, vom 9. 3. 1951, ISA, $2417 / 1$. 
daten an unserer Seite für die Verteidigung Europas dienen sollen. "11 Die Israelis sahen das ganz anders. In ihren Augen handelte es sich um Verbrecher, die vor allem Juden umgebracht hatten. Ihre Begnadigung bedeutete für sie, daß Judenmörder ungestraft davonkamen.

Adenauer und seine politischen Verbündeten unternahmen bedeutende Anstrengungen zur Freilassung ehemaliger Nationalsozialisten, die Kriegsverbrecher mit eingeschlossen. So nutzte der Kanzler den Besuch des amerikanischen Außenministers John Foster Dulles am 5. Februar 1953, um sich für die verurteilten Nationalsozialisten einzusetzen. ${ }^{12}$ Doch er war nicht der einzige. Dem im Spandauer Gefängnis in Berlin einsitzenden Albert Speer wurde unerklärlich viel Aufmerksamkeit sowohl von deutscher als auch von amerikanischer Seite zuteil. ${ }^{13} \mathrm{Die}$ einzelnen Kriegsverbrecherprozesse, die Entschädigung ehemaliger Nationalsozialisten, das Desinteresse der Justiz an mutmaßlichen NS-Verbrechern, die Freilassung von Nationalsozialisten auf Bewährung sowie die Freisprüche in solchen Prozessen wurden in Israel genau registriert. Auch das Auftauchen von Neonazis wurde in Israel zur Kenntnis genommen, doch der Kalte Krieg führte auch in Israel zu einer gewissen Abstumpfung in diesem Bereich.

Die strafrechtliche Verfolgung von Kriegsverbrechern in der Bundesrepublik war ein langsam vor sich gehender Prozeß. Angesichts der zahlreichen Anklagen wegen Verbrechen gegen das jüdische Volk ergab sich bald die Notwendigkeit eines geeigneten Verfahrens für die Einvernahme von Zeugen in Israel, und zwar in einer Form, die auch von deutschen Gerichte akzeptiert werden konnte. Die Israel-Mission in Köln übermittelte dem israelischen Generalstaatsanwalt regelmäßig Gesuche für Zeugenanhörungen, der wiederum die Polizei mit der Auffindung der Zeugen und der Durchführung der Befragungen beauftragte. Doch die deutschen Gerichte waren besonders an direkten Anhörungen interessiert. In Fällen, in denen die Einvernahme mehrerer Zeugen erforderlich war, schlugen die deutschen Gerichte vor, Untersuchungsrichter nach Israel zu entsenden, was von den israelischen Behörden aufgrund der fehlenden diplomatischen Beziehungen abgelehnt wurde. Da die israelische Seite die Verfolgung von NS-Verbrechern unterstützte, machte sie den Gegenvorschlag, solche Zeugenanhörungen durch einen israelischen Richter im Beisein eines deutschen Richters als Beobachter ohne Mitspracherecht durchzuführen. Der Generalstaatsanwalt des Staates Israel fügte hinzu, daß die Zeugenbefragung auf keinen Fall in deutscher Sprache stattfinden dürfe. ${ }^{14} \mathrm{Im}$ folgenden entwickelten sich verschiedene Verfahren, die so lange in

11 Memorandum von W.D. Allen vom 29. 1. 1951, PRO, FO 371/14226.

12 Protokoll vom 5. 2. 1953, PA, 270.

13 McCloy an Dulles vom 27.6. 1958; Dulles an McCloy vom 15.7. 1958; David Bruce an Dulles vom 31. 7. 1958, Amherst College Archives, McCloy Papers, Box GY2, Series 14, Folder 9.

14 Untersuchungsrichter am Landgericht Hamburg an die Israel-Mission in Köln vom 10. 11. 1954; Shinnar an den Generalstaatsanwalt vom 25.11. 1954; der Rechtsberater der Israel-Mission an Shinnar vom 16. 12. 1954; der Generalstaatsanwalt an den Rechtsberater des israelischen Außenministeriums vom 10.12. 1954; Shinnar an den Rechtsberater der Israel-Mission vom 23. 12. 1954; der Generalstaatsanwalt an Shinnar vom 13. 1. 1955, ISA, $578 / 20$. 
Kraft blieben, bis die deutschen Gerichte dazu übergingen, israelische Zeugen direkt in Deutschland zu vernehmen. In der Korrespondenz zu dieser Frage betonte Shinnar das israelische Interesse, die deutschen Anstrengungen zu unterstützen, um die Schilumim nicht zu gefährden. Die strafrechtliche Verfolgung von NSVerbrechern in der Bundesrepublik machte die sich aus den Nicht-Beziehungen ergebenden Komplikationen und die praktische Notwendigkeit formaler Beziehungen besonders deutlich.

\section{Die Verhaftung Eichmanns und die Vorbereitung des Prozesses}

Der Fall Eichmann verkörperte zahlreiche Aspekte der Kriegsverbrecherproblematik. Eichmann hatte während des Krieges die sogenannte Abteilung IV B 4 (Judenevakuierung) des Reichssicherheitshauptamtes geleitet. In dieser Funktion war er für die „Entjudung“ des deutschen Machtbereiches oder, mit anderen Worten, für die „Endlösung der Judenfrage“, d.h. für die Vernichtung des Judentums verantwortlich. Eichmann wurde nach dem Krieg verhaftet, blieb jedoch unerkannt und konnte entkommen. Eine Weile hielt er sich in Westdeutschland versteckt, und im August 1950 gelang ihm mit Hilfe einer Geheimorganisation von SS-Veteranen und eines kroatischen Ordens die Flucht nach Argentinien, wo er sich unter falschem Namen niederließ.

Eichmann war kaum bekannt und weckte deshalb in der Öffentlichkeit nur geringe Aufmerksamkeit. Er hatte das Rampenlicht der Öffentlichkeit stets gemieden, und selbst seine Fahnder hatten Schwierigkeiten, ein Bild von ihm zu finden. Dies dürfte mit ein Grund dafür gewesen sein, daß er in den Kriegsgefangenenlagern unerkannt geblieben war. Doch die unglücklichen Juden, die ihm während des Krieges in Berlin, Wien, Prag, Budapest, Mukacevo oder anderswo begegnet waren, kannten seine Aufgabe und seine "Leistungen" ausgezeichnet. Während sein Name bereits in den Nürnberger Prozessen auftauchte, wurde die israelische Öffentlichkeit erst 1955 auf Eichmann aufmerksam, als der aus Ungarn stammende Journalist Malkiel Grünwald einen führenden zionistischen Aktivisten aus Ungarn namens Rudolf Kastner öffentlich der Kollaboration mit den Nationalsozialisten zum Nachteil der Juden beschuldigte. Im folgenden Verleumdungsprozeß von Kastner gegen Grünwald, der starke Beachtung fand, wurde der Name Eichmann sehr häufig erwähnt. Eichmann wurde in Israel zum Inbegriff der „Endlösung“, zum Mephisto. Sein Name stand zuoberst auf der israelischen Liste der Kriegsverbrecher. Zahlreiche Stellen verpflichteten sich zur Suche nach Eichmann, bei manchen blieb es bei der Absichtserklärung. Es zirkulierten $\mathrm{Ge}-$ rüchte, wonach er in Ägypten, Kuwait und in verschiedenen südamerikanischen Staaten gesehen worden sei. Die meisten dieser Gerüchte stellten sich jedoch als falsch heraus und zeugen bestenfalls davon, wie stark Eichmann die Phantasie des israelischen Publikums beschäftigte. Die intensive Beschäftigung mit Eichmann in Israel erklärt auch die spontanen Gefühlsausbrüche und die weltweite Neugier, die dessen Auftauchen in Israel auslöste. 
Eichmann wurde nach dem Krieg zum ersten Mal 1957 in Argentinien aufgespürt. Der Staatsanwalt des Landes Hessen, Fritz Bauer, informierte darüber seinen Ministerpräsidenten Georg August Zinn sowie den Leiter der Israel-Mission Shinnar. Bauer befürchtete, die Information könnte durchsickern und Eichmann gewarnt werden. Der israelische Geheimdienst Mossad ging der Information nach, seine Nachforschungen förderten jedoch nichts zutage. Am 6. Dezember 1959 notierte Ben Gurion in sein Tagebuch, daß Eichmann in Argentinien gesehen worden sei, und daß er den Mossad anweisen werde, „sich der Sache anzunehmen". Der israelische Geheimdienst entführte den Mann sodann in Buenos Aires und schmuggelte ihn nach Israel. Am 22. Mai 1960 wurde er in Israel der Polizei übergeben. Am folgenden Tag informierte Ben Gurion die Knesset über die Verhaftung Eichmanns in Israel. Die Gefangennahme Eichmanns überraschte die israelische Öffentlichkeit, und die Nachricht verbreitete sich in Windeseile. Gefühle der Erleichterung und Stolz über die Leistung des Mossad machten sich breit. In der israelischen Presse hieß es, die Welle des Zorns sei nicht gegen Deutschland, sondern nur gegen Eichmann gerichtet. ${ }^{15}$ Altbundespräsident Heuss, der sich zur Zeit von Ben Gurions Erklärung zur Gefangennahme Eichmanns gerade zu Besuch in Israel aufhielt und die große Aufregung miterlebte, zeigte gegenüber Journalisten Verständnis für die Reaktion der Öffentlichkeit und erklärte, er sei überzeugt, daß Eichmann ein fairer Prozeß gemacht werde. ${ }^{16}$ Dies war die erste deutsche Reaktion - die eines Augenzeugen. Die Weltgemeinschaft war nicht weniger überrascht. Doch bei allem Verständnis für das Anliegen wurde die Entführung als Akt internationaler Piraterie betrachtet. Argentinien reichte eine Klage im UNO-Sicherheitsrat ein, und Israel stellte klar, daß der Gefangene an keinen anderen Staat ausgeliefert werde. Andererseits versuchte die israelische Regierung, Argentinien zu beschwichtigen. Bei einem Empfang anläßlich des Besuchs von Argentiniens Präsident Arthuro Frondisi in Bonn auf dem Höhepunkt der Aufregung rund um die Entführung Eichmanns meinten die anwesenden deutschen Gäste, einschließlich Erhard, gegenüber dem ebenfalls anwesenden Shinnar, Israel solle hart bleiben.

Die israelische Polizei richtete eine spezielle Ermittlungsabteilung - die sogenannte Abteilung 06 - ein und besetzte sie mit deutschsprachigen und besonders erfahrenen Beamten, in deren Familien niemand im Holocaust umgekommen war. Die Behörden fürchteten, einzelne Beamte könnten versuchen, persönlich Rache an Eichmann zu üben. Die Arbeit der Ermittler glich immer mehr dem Studium von Geschichtsstudenten, die sich eingehend mit dem Holocaust befaßen. Sie durchforsteten zahlreiche Archive in Israel, Westdeutschland, USA, Frankreich und in anderen Ländern und forderten bei fremden Regierungen Beweismaterial an. Von den osteuropäischen Ländern reagierten die Tschechoslowakei, Polen, Ungarn und Rumänien positiv. Die bulgarische Regierung gab an, über keine Dokumente zu verfügen, und von der Sowjetunion kam gar keine Antwort. Die israelischen Ermittler verzichteten auf eine Anfrage bei den Behörden der DDR und begründeten dies mit den fehlenden Beziehungen zwischen beiden Staaten.

15 HaAretz (Tel Aviv); Yedioth ACHRONOTH; MA'ARIV, jeweils 24.-25. 5. 1960.

16 HAAreTz ('Tel Aviv) vom 24. 5. 1960. 
Ostdeutschland stellte Israel später aus eigener Initiative im Rahmen ihrer Propagandakampagne Dokumente zur Verfügung. Die Anklage gegen Eichmann leiteten Generalstaatsanwalt Gideon Hausner und Staatsanwalt Gabriel Bach. ${ }^{17}$

Probleme bereitete die Übernahme der Verteidigung. Trotz ausdrücklicher Genehmigung der Anwaltskammer war kein israelischer Anwalt bereit, die Verteidigung Eichmanns zu übernehmen. Schließlich akzeptierte der deutsche Anwalt Robert Servatius das Mandat. Die Nominierung von Servatius, der bereits in den Nürnberger Prozessen Erfahrungen gesammelt hatte, war nicht unumstritten. Offiziell wurde erklärt, Servatius sei von Eichmanns Familie beauftragt worden, doch kritische Stimmen behaupteten, in Wirklichkeit stünden neonazistische Kreise dahinter. Die israelischen Behörden prüften Servatius' Vergangenheit und betonten die Bedeutung der Wahl eines angesehenen Rechtsanwalts angesichts der großen weltweiten Aufmerksamkeit, die dieser Prozeß weckte. ${ }^{18} \mathrm{Um}$ die Vertretung vor Gericht durch einen ausländischen Anwalt zu ermöglichen, war eine Gesetzesänderung nötig. Schließlich stand Servatius auch ein israelischer Anwalt zur Seite. Obwohl anfänglich erklärt wurde, die Kosten der Verteidigung würden von Eichmanns Familie getragen, kam schließlich der israelische Steuerzahler dafür auf. ${ }^{19}$ Die Bundesrepublik lehnte ein Finanzierungsgesuch von Servatius ab. Auch die zahlreichen anderen Probleme konnten bis zur Eröffnung des Prozesses am 11. April 1961 zumeist gelöst werden.

Ein weiterer umstrittener Punkt was das Gerichtsverfahren selbst und die Besetzung des Gerichts. Es gab Stimmen, die bedauerten, daß die Entführer Eichmann nicht auf der Stelle liquidiert hatten, wodurch man sich ihrer Ansicht nach viele Probleme erspart hätte. Andere, darunter auch führende Persönlichkeiten wie Nahum Goldmann und der ehemalige Präsident des American Jewish Committee (AJC), Joseph M. Proskauer, forderten, das Gericht mit Richtern verschiedener Nationalität zu besetzen oder wenigstens einen ausländischen Gerichtspräsidenten zu ernennen. ${ }^{20}$ Zudem wurden Vorbehalte zum Gerichtsort Jerusalem laut und ein neutraler Ort gefordert, um die absolute Unparteilichkeit des Gerichts sicherzustellen. Einige Beobachter befürchteten einen Anstieg des Antisemitismus als Folge des Eichmannprozesses. All diese Einwände beruhten mehrheitlich auf dem britischen Rechtsgrundsatz, wonach man Gerechtigkeit nicht nur schaffen, sondern auch zeigen müsse. Ben Gurion und die Mehrheit der israelischen Öffentlichkeit wiesen die Einwände zurück. Die Kritiker stellten Objektivität, Fairneß und Unparteilichkeit der israelischen Richter in Frage und mußten sich dafür den Vorwurf gefallen lassen, auch deren Aufrichtigkeit und Integri-

17 ASHMAN/WAGMAN, Tsayade ha nazim; BAR-ZOHAR, B'yom nakam; HAREL, Das Haus in der Garibaldistrasse; WIESENTHAL, Recht, nicht Rache; MAN/DAN, Eichmann be-yaday; Perlmann, Eich nitpas eichman; The CaSe Against AdOLF EichmanN; Robinson, And the Croocked; Baum, Christliche Endlösung; MisHPAT EICHMANN; HAUSNER, Gerechtigkeit.

18 Knesset-Protokolle [hebr.], 173. Sitzung vom 15.11. 1960, S. 209 und 183. Sitzung vom 29. 11.1960 , S. 345.

19 Knesset-Protokolle [hebr.], 295. Sitzung vom 3. 1. 1961, S. 630-631.

20 ZwISCHEN MORAl UND REAlPolitik, Dok. Nr. 179, S. 519-520; Dok. Nr. 181, S. 524 526. 
tät in Zweifel zu ziehen, was als Affront gegen die israelische Justiz empfunden wurde. Ben Gurion bestand auf der Durchführung des Prozesses in Israel. Es war eine einmalige Gelegenheit für ein verfolgtes Volk, mit einem Peiniger abzurechnen. Der israelische Ministerpräsident betrachtete den Prozeß gegen Eichmann zudem als Gelegenheit, der israelischen Jugend den Schrecken des Holocaust und der Diaspora zu vermitteln und ihr eine Lektion über den Zionismus zu erteilen bzw. sie über den Wert eines eigenen Staates aufzuklären. Der Welt wollte er die Verbrechen gegen die Juden deutlich vor Augen halten. ${ }^{21}$ Für diese Aufgabe eigneten sich in Ben Gurions Augen nur jüdische Richter. Diese Gelegenheit wollte er auf keinen Fall verpassen. Ein weiterer - selten genannter - Grund für das israelische Beharren, Eichmann im eigenen Land den Prozeß zu machen, war der Umstand, daß in Israel für solche Verbrechen die Todesstrafe verhängt werden konnte. Die Regierung entschied sich für die Durchführung des Eichmannprozesses in Israel.22

Das für den Prozeß vorgesehene Bezirksgericht Jerusalem wurde von Richter Benjamin Halevy präsidiert, der auch die Verhandlungen in der Verleumdungsklage gegen Kastner geleitet hatte. Doch Ben Gurions Mapai-Partei mißtraute Halevys Unvoreingenommenheit und veranlaßte die Verabschiedung eines Gesetzes, wonach Prozesse, in denen die Todesstrafe gefordert wird, von Richtern des Obersten Gerichtshofs zu leiten seien. ${ }^{23}$ Halevi wurde seiner Funktion als Gerichtsvorsitzender enthoben und mußte sich in ein Gremium von drei Richtern einfügen. Das neue Gesetz sah zudem vor, daß bei Fällen, in denen die Todesstrafe gefordert wird, keine Zivilklagen hinzugefügt werden dürfen. Diese scheinbar vernünftige Bestimmung war in Wirklichkeit dazu bestimmt, den Auftritt des ostdeutschen Rechtsanwalts Friedrich Kaul als Privatkläger im Eichmannprozeß und die damit verbundene Gefahr der Verwandlung des Prozesses in eine Plattform für DDR-Propaganda vor allem gegen die Bundesrepublik zu verhindern.

\section{West- und ostdeutsche Initiativen bis zum Prozessbeginn}

Einen Tag nach der Verlautbarung Ben Gurions nahm das Auswärtige Amt in Bonn eine erste Lagebeurteilung vor: Die Chancen für eine Auslieferung Eichmanns wurden als äußerst gering eingestuft, da kein entsprechendes bilaterales Abkommen existierte. Israel werde Eichmann auf Grund des Gesetzes für die Verfolgung von Nationalsozialisten und deren Helfer (1950) den Prozeß machen, das auch die Todesstrafe vorsehe. Der Verfasser meinte zudem, die Bundesrepublik dürfe die Kosten der Verteidigung nicht übernehmen - diese werde letztlich der Staat Israel tragen -, doch Bonn solle Israel juristischen Beistand leisten und einen Beobachter $\mathrm{zu}$ den Gerichtsverhandlungen schicken. ${ }^{24}$ So sollte es auch kommen. Entsprechend informierte das Auswärtige Amt alle westdeutschen Ver-

21 Ben Gurion an Galili vom 26. 5. 1960, BGA, Correspondence File, 1960.

22 Dawar (Tel Aviv) vom 27.6.1960.

23 KNESSET-Protokolle [Original hebr.], 4. Knesset, 222. Sitzung am 31. 1. 1961.

24 Raab an den Staatssekretär vom 24. 5. 1960, PA, 708, 81.07, 92.19, Bd. 2. 
tretungen in den arabischen Staaten. Aus diesen Botschaften ging im wesentlichen folgendes hervor: Die Bundesrepublik unterstützte den jüdischen Staat bei seinen Vergeltungsanstrengungen und werde dies auch in Zukunft tun. Die Bundesrepublik werde die Auslieferung Eichmanns nicht beantragen und distanziere sich von diesem Mann. Die Bundesrepublik sei besorgt über die arabischen Reaktionen.

Im Bewußtsein der israelischen Weigerung, Eichmann in einem anderen Land vor Gericht zu stellen, erwogen die westdeutschen Behörden die Möglichkeit, die Auslieferung Eichmanns im Falle seiner Rückführung nach Argentinien zu fordern. Eichmann besaß die argentinische Staatsbürgerschaft und war nach argentinischem Recht unschuldig. ${ }^{25}$ Jüdische und israelische Beobachter hielten ein westdeutsches Auslieferungsgesuch an Israel für sehr unwahrscheinlich, nicht nur wegen der fehlenden Auslieferungsvereinbarung, sondern auch deshalb, weil von israelischer Seite angenommen wurde, daß ein solcher Prozeß der Bundesrepublik sehr ungelegen käme, da er die ehemaligen Nationalsozialisten in den obersten Bundesbehörden ins Rampenlicht rücken könnte. ${ }^{26}$ Eine andere Haltung nahm Eichmanns deutscher Verteidiger Servatius ein. Er versuchte, den Prozeß in die Bundesrepublik zu verlagern mit der Begründung, daß sein Mandant als deutscher Staatsbürger ein Anspruch auf Schutz habe und somit in Deutschland vor Gericht $\mathrm{zu}$ stellen sei. Zudem erinnerte er die westdeutschen Behörden daran, daß in Deutschland die Todesstrafe abgeschafft sei. ${ }^{27}$

In einem Rechtsgutachten für den Bundesminister wurde die Auffassung vertreten, eine Freilassung oder Auslieferung stehe nicht zur Diskussion. Es gebe zwischen der Bundesrepublik und Israel kein Auslieferungsabkommen, und ein Auslieferungsgesuch könne man nicht mit der drohenden Todesstrafe begründen. Zudem sei die Einmischung in die Gesetzgebung eines anderen Staates nicht angebracht. Die israelische Interpretation des Grundsatzes "Nulla poena sine lege“ rechtfertige jedenfalls keine Intervention. Die westdeutschen Behörden waren offensichtlich bemüht, sich so wenig wie möglich für Eichmann einzusetzen. ${ }^{28}$ Servatius versuchte es noch auf anderen Wegen: Er forderte zunächst in einer Eingabe an das Auswärtige Amt unter Hinweis auf den Staatssekretär im Bundeskanzleramt, Hans Globke, die Freilassung seines Mandanten. ${ }^{29}$ Hierauf erhielt er jedoch ebenso wenig eine Antwort wie auf eine spätere Eingabe, in der er einen „diplomatischen Schutzanspruch" seines Mandanten postulierte, der vor einem Verwaltungsgericht geltend gemacht werden konnte und die Bundesrepublik dazu gezwungen hätte, Eichmanns Verteidigung zu stellen. ${ }^{30}$

25 Marmann an den Bundesminister über den Staatssekretär vom 20. 6. 1960, PA, 708, 82.70, 92.19, Bd. 2.

26 Ralph Friedmann an die Mitglieder des Auswärtigen Ausschusses vom 19. 7. 1960, YIVO, AJC, FAD-1, Box 26, Germany, West.

27 Servatius an das AA vom 5.2. 1960; Marmann an den Bundesjustizminister vom 14. 2. 1960; Dollinger, Bundesjustizministerium, an das AA vom 28. 2. 1960, PA, 708, 82.70, 92.19 , Bd. 2.

28 Marmann an den Bundesminister über den Staatssekretär vom 3. 3. 1960, PA, 708, 82.70, 92.19, Bd. 2.

29 Servatius an das AA vom 5. 2. 1960, PA, 708, 82.70, 92.19, Bd. 2.

30 Preuschen an das AA vom 14. 4. 1961, PA, L3, 80.00, 508/61, Bd. 7. 
Die mehrköpfige Delegation, die die Bundesrepublik Deutschland zum Prozeß gegen Eichmann nach Israel entsandte, setzte sich aus dem offiziellen Beobachter Gerhard von Preuschen und seinem Stellvertreter, einem jungen Rechtsanwalt, einem Beamten des Presse- und Informationsamtes, einem Wissenschaftler, einem Verwaltungsdienst- und Konsularsekretär sowie Sekretärinnen und Kodierungsspezialisten zusammen. Inoffiziell gehörte der westdeutschen Delegation auch der Journalist Rolf Vogel an. ${ }^{31}$ Von Preuschen war ein Freund des Bundesaußenministers und von ihm persönlich mit dieser Aufgabe betraut worden. Er hatte an der Verschwörung des 20. Juli 1944 teilgenommen, und seine Vergangenheit galt als unbefleckt. Bei den anderen Delegationsmitgliedern handelte es sich um Vertreter der Nachkriegsgeneration. Vogel behauptete später, Bundeskanzler Adenauer habe ihn zu seinem persönlichen Beobachter ernannt. ${ }^{32}$

Das israelische Außenministerium stellte der deutschen Delegation Privatwohnungen zur Verfügung, kümmerte sich um ihre logistischen Bedürfnisse und reservierte für sie Plätze in der vordersten Reihe im Beit Ha'Am-Saal („Volkshaus“), wo der Prozeß stattfand. Bei ihrer Ankunft wurden der Delegation Leibwächter zugeteilt, die sich jedoch schon bald als unnötig erwiesen, deshalb abgezogen und erst bei der Bekanntgabe des Gerichtsurteils wieder eingesetzt wurden. Die Deutschen wurden freundlich empfangen, pflegten während ihres Aufenthalts gesellschaftlichen Verkehr mit israelischen Vertretern und schienen in keiner Weise bedroht. Nur einmal erlebte die Delegation eine von der kommunistischen Partei organisierte, feindselige Demonstration gegen Eichmann, die indirekt auch gegen die Bundesrepublik gerichtet war. Die Mitglieder der deutschen Delegation trafen sich mehrmals mit Vertretern des israelischen Außenministeriums, besonders mit Max Varon von der Westeuropaabteilung und mit Chaim Yachil. Von Preuschen traf den israelischen Justizminister, den in Deutschland geborenen Pinchas Rosen, und Vertreter der Anklage.

Die bundesdeutsche Delegation hatte verschiedene Aufgaben. Von Preuschen lieferte ab 5. April 1961 ausführliche Berichte über die Gerichtsverhandlungen, über seine offiziellen Kontakte mit israelischen Vertretern und sporadisch auch über prozeßbegleitende Ereignisse und Umstände sowie über Israel im allgemeinen. Vogel, der über zahlreiche Kontakte in Israel verfügte, berichtete sowohl über Ereignisse im Zusammenhang mit dem Prozeß als auch losgelöst davon. ${ }^{33}$ Zusammen mit dem Delegierten des Presse- und Informationsamtes informierte er zudem über die israelische Presselandschaft. Die deutsche Delegation und die Israel-Mission in Köln kümmerten sich um die Beschaffung von Dokumenten und schriftlicher Zeugenaussagen durch deutsche Gerichte sowie um die Vorladung von Zeugen aus Deutschland. Als bekannt wurde, daß sich eine westdeutsche Delegation in Jerusalem aufhielt, wurde sie von der Bevölkerung mit Memoranden, Petitionen und allen möglichen Bitten überhäuft, die alle ordnungsgemäß nach Bonn weitergeleitet wurden. Die Generalstaatsanwaltschaft des Landes Hessen schickte den Staatsanwalt Dietrich Zeug, der jüdischer Abstammung war,

31 Vermerk der deutschen Delegation vom 29. 3. 1961, PA, 708, 82.70, 92.19.

32 Der Deutsch-Israelische Dialog, S. 184-186.

33 Ebd. 
nach Jerusalem, um dort die Landesjustizverwaltung Ludwigsburg zu vertreten und die Entsendung eines offiziellen Beobachters der DDR zu verhindern. ${ }^{34}$ Einundfünfzig deutsche Journalisten wohnten der Eröffnung des Prozesses bei. Die SPD und andere Organisationen entsandten eigene Beobachter, und das Auswärtige Amt beschäftigte getarnte PR-Agenten in Jerusalem..$^{35}$

Friedrich K. Kaul, ein ostdeutscher Rechtsanwalt jüdischer Abstammung, kam nach Jerusalem, um sich der Anklage gegen Eichmann mit Einzelklagen anzuschließen. ${ }^{36}$ Doch wie wir gesehen haben, hatte die israelische Regierung vorsorglich Schritte unternommen, um genau dies zu verhindern. Die israelischen Behörden verweigerten dem ostdeutschen Rechtsanwalt die Anerkennung als offizieller Vertreter, so daß er gezwungen war, nach Ablauf der Aufenthaltsbewilligung wieder auszureisen und ein neues Einreisevisum zu beantragen. Kaul war mit Beweismaterial angereist, das er direkt der Anklage vorlegte. Anläßlich einer Unterredung mit dem israelischen Justizminister verlangte er, als offizieller Vertreter anerkannt zu werden, was jedoch mit dem Hinweis auf seine ostdeutsche Staatsbürgerschaft und die fehlenden Beziehungen zwischen Israel und der DDR abgelehnt wurde. Das Verhalten der DDR und das Bewußtsein um die internationale Bedeutung von Kauls Mission erfüllten die Israelis zwar mit Hoffnung, doch insgesamt schien die israelische Politik eher vom freundschaftlichen Verhältnis zur Bundesrepublik geprägt, die Kauls Mission als Gefahr für ihr Ansehen betrachtete. Man gewinnt den Eindruck, daß beide Regierungen Kaul gemeinsam Hindernisse in den Weg stellten. Dazu ein Beispiel: Kaul war gezwungen, eine zuvor angekündigte Pressekonferenz abzusagen, nachdem ihm die Benutzung des für solche Anlässe üblichen Raums im israelischen Presseclub verweigert wurde, und mußte mit Räumlichkeiten vorliebnehmen, die ihm die kommunistische Partei zur Verfügung stellte. ${ }^{37}$ Die Eröffnung des Eichmannprozesses wurde von sieben ostdeutschen Journalisten mitverfolgt, die kurz darauf wieder abreisten. Die meisten von ihnen waren jüdischer Abstammung und mit der offiziellen Linie der DDR-Medien offensichtlich im Zwiespalt.

Die Entführung Eichmanns wurde in der Bundesrepublik mit Unbehagen aufgenommen. Die Früchte jahrelanger Imagepflege vor allem gegenüber Israel, aber auch im europäischen und globalen Kontext schienen durch den bevorstehenden Prozeß gefährdet. Am 21. Februar 1961 erteilte von Brentano den deutschen Vertretungen im Ausland Instruktionen die politische Öffentlichkeitsarbeit zum Fall Eichmann betreffend. Der Bundesaußenminister rechnete darin mit einem Ausbruch von Deutschfeindlichkeit sowie mit einer Hetzkampagne des Ostblocks, insbesondere der DDR, gegen die Bundesrepublik. Die westdeutschen Auslandvertretungen wurden aufgefordert, in einer Gegenkampagne deutlich zu machen, $\mathrm{da} ß$ die Bundesrepublik daran interessiert sei, die NS-Verbrecher zu bestrafen und

34 Marmann, Notiz über die Besprechung mit Grützner vom 12.1. 1961, PA, 708, 82.70, 92.19, Bd. 2.

35 Ahrens an den Beobachter in Jerusalem vom 26. 5. 1961, PA, L3, 80.00, 508/61, Bd. 2.

36 KAUL, Der Fall Eichmann.

37 Von Preuschen an das AA vom 23. 6. 1961; Stercken an das BPA und das Bundeskanzleramt über das AA vom 21. 6. 1961, PA, L3, 80.00, 508/61, Bd. 7. 
bereits vieles getan habe, um das Unrecht wiedergutzumachen. In Anlehnung an ein Zitat von Golda Meir wurde zudem betont, daß die Bürger der Deutschen Demokratischen Republik die gleiche Verantwortung für die Vergangenheit trügen, bisher aber keine Wiedergutmachung an NS-Opfer geleistet hätten. Die Machthaber der sowjetisch besetzten Zone, schrieb Bundesaußenminister von Brentano, setzten Hitlers Diktatur mit denselben Methoden und zum Teil denselben Personen fort, während im Gegensatz dazu die Politik der Bundesregierung das Vertrauen auch der israelischen Regierung gefunden habe. Von Brentanos Ausführungen enthielten eine Darstellung der Geschichte, die mindestens zum Teil fragwürdig war: Die Deutschen seien die ersten Opfer des Nationalsozialismus gewesen. Deutsche hätten zweimal versucht, Hitler zu töten, und die Wahrheit über die Vorgänge des Krieges sei dem deutschen Volk erst nach Kriegsende bekannt geworden. ${ }^{38}$ Die Missionen wurden aufgefordert, die öffentliche Meinung und die Presse in den jeweiligen Ländern zu verfolgen und darüber zu berichten. Das Auswärtige Amt versorgte die Vertretungen im Ausland mit Unterlagen für die Öffentlichkeitsarbeit, die Statistiken über Verfolgungen und Entschädigung, Informationen über Nationalsozialisten in der DDR sowie Berichte über Israel und über die deutsch-israelischen Beziehungen enthielten. ${ }^{39}$ Ferner stellte das Amt Bücher und Filme über Israel zur Verfügung bzw. veranlaßte die Produktion solcher Filme, zum Ärger der Araber.

In einem Schreiben an Bundeskanzler Adenauer vom 10. März 1961 zeigte sich von Brentano besorgt über die bevorstehenden Entwicklungen. Die Eröffnung des Prozesses gegen Eichmann war etwa auf das Datum der Ankunft Adenauers in den Vereinigten Staaten angesetzt. Der USA-Besuch des Kanzlers „fällt mit dem Beginn dieses makabren Schauspiels zusammen", wie sich von Brentano ausdrückte..$^{40} \mathrm{Im}$ Bewußtsein der überragenden Rolle, die der Kanzler dem amerikanischen Judentum nach wie vor beimaß, riet ihm von Brentano zu vorbeugenden Schritten, um die amerikanische Presse und die amerikanisch-jüdische Öffentlichkeit zu beschwichtigen, eventuell durch einen Auftritt im amerikanischen Radio und Fernsehen. „Wir werden uns aus Anlaß dieses schaurigen Prozesses eindeutig distanzieren müssen", stellte der Bundesaußenminister fest. ${ }^{41}$ Der Botschafter der Bundesrepublik Deutschland in Washington schrieb in einer Aufzeichnung an das Auswärtige Amt, die Bundesrepublik zweifle nicht an Israels Recht, Eichmann vor Gericht zu stellen, solange es sich nicht um einen Schauprozeß handle.42 In einer Fernsehansprache am 11. April 1961 betonte der Bundeskanzler den Unter-

38 Von Brentano an die deutschen Vertretungen im Ausland vom 21. 2. 1961, PA, L3, 80.00, 508/63, Bd. 4.

39 Ebd.; Dr. Dallinger, Bundesministerium für Justiz, an die Zentrale Rechtsschutzstelle des AA vom 24. 2. 1961, PA, 708, 82.70, 92.19; Übersicht über die Verfolgung nationalsozialistischer Straftaten in der BRD, PA, L3, 80.00, 508/61, Bd. 5; Botschaft Bern an das AA vom 4. 3. 1961, PA, L3, 80.00, 508/61, Bd. 4; Die Botschaft in Tel Aviv an die Abt. Levante in London vom 23. 2. 1961, PRO, FO 371/159104.

40 Von Brentano an Adenauer vom 10. 3. 1961, BArch, N 1239/158, Nr. 179.

41 Ebd.

42 Die deutsche Botschaft in Washington an das AA vom 13. 3. 1961, PA, L3, 80.00, 508/61, Bd. 4. 
schied zwischen dem Deutschland der Vergangenheit und der Gegenwart und wies quasi zur Veranschaulichung auf seine Freundschaft mit Ben Gurion hin. ${ }^{43}$ Er unterstrich zudem das Recht Israels, Eichmann vor Gericht zu stellen. Das Auswärtige Amt versuchte für seine Öffentlichkeitsarbeit auch den SPD-Vorsitzenden Willy Brandt zu gewinnen. ${ }^{44}$ Die Israelis ihrerseits erwogen, Bonn mit einer offiziellen Erklärung zu beschwichtigen ${ }^{45}$, was auf den starken Willen in Israel hindeutet, alles zu unterlassen, was die guten Beziehungen zur Bundesrepublik gefährden könnte. Generalstaatsanwalt Hausner sprach vor Gericht auf Anregung Ben Gurions nur von „Nazis" und mied das Wort "Deutsche“. Auch in der Bundesrepublik schien mit dem Eichmannprozeß ein empfindlicher Nerv getroffen, wie ein „Aufruf“ des „Bundes der Opfer des Faschismus" zeigt, der unter anderem vom ehemaligen Münchener Bürgermeister Scholl und von Schenk Graf von Stauffenberg unterzeichnet wurde. 46

Die Bundesregierung entschloß sich zu Überzeugungskampagnen im In- und Ausland. Demnach sollte der eigenen Bevölkerung vor Augen geführt werden, daß Unrecht im Namen Deutschlands geschehen sei und daß Eichmann dafür Mitverantwortung trage, wodurch sich das Recht Israels ergebe, diesen Mann vor Gericht zu stellen. Nicht die Frage der kollektiven Verantwortung, sondern die Verbrechen selbst standen im Mittelpunkt, wenn auch eingeräumt wurde, daß es das deutsche Volk gewesen sei, das Hitler an die Macht gebracht habe. Während es also im eigenen Land vor allem darum ging, die Bevölkerung behutsam über unbequeme Tatsachen aufzuklären, erforderte die Situation im Ausland genau das Gegenteil, nämlich die Versöhnungsleistungen der Bundesrepublik hervorzuheben, um den Ausbruch antideutscher Gefühle zu vermeiden. Während viele deutsche Bürger am liebsten einen Schlußstrich unter die belastende Geschichte gezogen hätten, weckte der Eichmannprozeß bei den Opfern der deutschen Besatzung und den Augenzeugen von NS-Greueln äußerst bittere Erinnerungen. Die westdeutschen Vertretungen im Ausland versuchten, die bisher gegen Kriegsverbrecher geführten Prozesse und die Hilfe für NS-Opfer in der Bundesrepublik hervorzuheben, um die gegenseitige Verständigung und Zusammenarbeit zwischen der Bundesrepublik Deutschland und dem Staat Israel zu unterstreichen. ${ }^{47}$ Das humanistische Antlitz der Nachkriegsdeutschen, die sich ihrer Vergangenheit gestellt und daraus gelernt hätten, sollte betont werden. Im Ausland standen sich

43 Von Brentano an die deutschen Vertretungen im Ausland vom 21. 6. 1961, PA, L3, 80.00, 508/63, Bd. 4; Vermerk über die Öffentlichkeitsarbeit der Bundesregierung im Zusammenhang mit dem Eichmannprozeß vom Juni 1961, PA, L3, 80.00, 508/63, Bd. 4; Hille an den Bundesminister vom 9. 3. 1961, PA, L3, 80.00, 508/63, Bd. 5.

44 Die deutsche Botschaft in Washington an das AA vom 13.3. 1961, PA, L3, 80.00, 508/61, Bd. 4.

45 Teddy Kolek an Shinnar vom 14. 3. 1961, ISA, 4318/5.

46 Aufruf des „Bundes der Opfer des Faschismus" vom 21. 3. 1961, LBI, Nachlaß Kurt R. Grossman, box 23, file 1 .

47 Dr. Gawlik an die Botschaft in Mexiko vom 14. 3. 1961, PA, 708, 82.70, 92.19; Federer, New York, an das Bundespresseamt vom 15. 3. 1961, PA, 708, 82.70, 92.19; die Botschaft in Washington an das AA vom 15.3. 1961, PA, L3, 80.00, 508/63, Bd. 4; Blankenhorn, Paris, an das AA vom 10. 4. 1961, PA, L3, 80.00, 508/63, Bd. 3. 
Antisemitismus und Antigermanismus gegenüber. Die Aufgabe der westdeutschen Diplomatie war es, beide zu neutralisieren.

Während die Bundesregierung versuchte, Staat und die Gesellschaft im Ausland möglichst positiv darzustellen, nahm der Ostblock und besonders die DDR die Rolle des Störenfrieds ein. Die Entführung Eichmanns und der nachfolgende Prozeß waren für den weltweit geführten Propagandakrieg, an dem sich die kommunistischen Parteien und deren Mitläufer führend beteiligten, ein Geschenk des Himmels. Ein bevorzugtes Zielpublikum waren die über die Welt verstreuten jüdischen Gemeinden. Doch auch in Israel fiel die kommunistische Propaganda in diesem Zusammenhang angesichts der latenten Deutschfeindlichkeit und den im Prozeß zutage geförderten Greueln teilweise auf fruchtbaren Grund.

\section{Die Debatte über Hans Globke im Rahmen des Eichmann-Prozesses}

Im Mittelpunkt der östlichen Propaganda stand Hans Globke. Die Erwähnung verschiedener ehemaliger SS-Offiziere und prominenter Nationalsozialisten im Eichmannprozeß, besonders die Enthüllung der Tatsache, daß diese unbehelligt in der Bundesrepublik lebten, rückte Globke noch stärker ins Rampenlicht. Hans Globke, Staatsekretär im Bundeskanzleramt und enger Mitarbeiter des Kanzlers, hatte als Ministerialrat im Reichsinnenministerium Kommentare zu den sogenannten Nürnberger Gesetzen sowie verschiedene andere antijüdische Verordnungen verfaßt. Zudem wurde ihm die Beteiligung an der Verfolgung slowakischer und griechischer Juden vorgeworfen. Globke brachte zu seiner Verteidigung vor, er sei nie Mitglied der NSDAP gewesen und habe mit seinen Kommentaren versucht, die Auswirkungen der NS-Gesetzgebung für die Juden abzumildern. Ferner bestritt er jegliche Beteiligung an der Verfolgung von Juden im Ausland. Globke, ein fleißiger Beamter mit ausgezeichnetem Erinnerungsvermögen, genoß das volle Vertrauen des alternden Kanzlers, der dringend auf seine Dienste angewiesen war. ${ }^{48}$

Die nicht gerade ruhmreiche Vergangenheit sowie die neue Machtfülle und Nähe zum Bundeskanzler machten Globke zum vortrefflichen Ziel für Attacken der Linken. Im Verlaufe des Eichmannprozesses wurde der Fall Globke unverhältnismäßig aufgebauscht. Unter den gegebenen Bedingungen wurde er vom Kanzler und der Bundesregierung jedenfalls in Schutz genommen. Die israelische Regierung beschloß mit der Bundesregierung zu kooperieren, die ihrerseits fest entschlossen war, die Verwicklung Globkes in den Eichmannprozeß um jeden Preis zu vermeiden, weniger um seinetwillen, sondern aus staatspolitischen Erwägungen.

Die israelische Strategie im Eichmannprozeß schloß auch die Verteidigung Globkes mit ein. Irgendwann im Verlaufe der Vorbereitungen zum Prozeß wurde

48 Det StaAtssekretãr Adenauers; Reinhardt, Der Fall Globke; Doktor Hans GLOBKE; GLOBKE UND DIE AUSROTTUNG DER JUDEN. 
die Entscheidung getroffen, das Verfahren nur auf Eichmann zu konzentrieren: Eichmann sollte als Inbegriff des Nationalsozialismus dargestellt und andere Themen gemieden werden. Mehrere Anhaltspunkte sprechen für Ben Gurions Teilnahme an den diesbezüglichen internen Besprechungen, deren Protokolle immer noch unter Verschluß gehalten werden. ${ }^{49}$ Das lakonische Leitmotiv des bevorstehenden Gerichtsverfahrens lautete „Eichmann und das jüdische Volk“. Alle anderen ehemaligen Nationalsozialisten waren somit von vornherein vom Verfahren ausgeschlossen. Gerechtfertigt wurde dies damit, daß diese entweder nicht mehr am Leben oder für die israelische Justiz unerreichbar waren bzw. sich weigerten, vor einem israelischen Gericht zu erscheinen. Versuche, deutsche Zeugen zur Aussage im Verfahren gegen Eichmann zu bewegen, scheiterten unter anderem an der Furcht der in Frage kommenden Zeugen, selbst verhaftet zu werden, trotz Immunitätsgarantien der israelischen Regierung. Unter diesen Umständen wäre die Beschäftigung mit Globke zwangsläufig eine unwillkommene Ablenkung gewesen. Die israelischen und westdeutschen Interessen stimmten in dieser Frage überein, und beide Regierungen sorgten gemeinsam dafür, Globkes Name vom Verfahren gegen Eichmann möglichst fernzuhalten.

Beim einzigen bisher gefundenen Dokument in dieser Angelegenheit handelt es sich offenbar um die Aufzeichnung eines diskreten Gesprächs zwischen Shinnar und einer oder mehrerer ungenannter Personen über G. (wahrscheinlich Globke), E. (Eichmann), Servatius, die Israelis und einen Geheimdienst, wobei aus dem Zusammenhang zu schließen ist, daß es sich dabei um den Bundesnachrichtendienst handelte. Globke war nicht anwesend, seine Teilnahme war aber offenbar erwartet worden. Die Gesprächsteilnehmer berieten darüber, wie die Hereinziehung von $\mathrm{G}$. in den E.-Prozeß verhindert werden könne. ${ }^{50}$ Dieses Ziel verfolgten auch der evangelische Theologie Heinrich Gruber, der in seiner Zeugenaussage im Eichmann-Prozess Globke positiv erwähnte, ${ }^{51}$ und der Generalstaatsanwalt, von dem von Preuschen zu berichten wusste, dass er Globke gegenüber absolut loyal sei. ${ }^{52}$

In der hitzigen Debatte vom Frühjahr 1965 über die deutschen Waffenlieferungen an Israel behauptete der Chefredakteur des Stern, Henry Nannen, der Staat Israel habe sich dazu verpflichtet, nichts zu unternehmen, was dem Ansehen der Bundesrepublik schaden könne und daher darauf verzichtet, im Verfahren gegen Eichmann eine Aussage Globkes zu erzwingen. Dies seien die Bedingungen für

49 Bericht v. Preuschens an das AA über die Besprechung mit Yachil vom 24. 5. 1961, PA, L3, $80.00,508 / 63$, Bd. 6 .

50 Aufzeichnung über das Gespräch mit Dr. Shinnar in Zürich vom 2. 11. 1960, ISA, 4327/12; ZwISCHEN MORAL UND REALPOLITIK, S. 539.

51 Von Preuschen an das Bundeskanzleramt vom 16. 5. 1961, PA, PA, L3, 80.00, 508/63, Bd. 4; von Preuschen an das AA vom 16. 5. 1961, PA, 1038, Bd. II, 708, 82.70, 92.19.

52 Von Preuschen an das AA vom 12. 5. 1961, PA, 1038, 708, 82.70, 92.19, Bd. 2. Hanna Yablonka behauptet in ihrer kürzlich erschienenen Arbeit über den Eichmannprozeß, daß es sich bei der Verständigung zwischen beiden Seiten in Sachen Globke um ein "Gentleman's Agreement" gehandelt habe. Irgendwelche anderen bundesdeutschen Gesten, wie zum Beispiel Waffenlieferungen, erwähnt sie nicht: YABLONKA, Israel gegen Eichmann, S. 65. 
das Rüstungsgeschäft gewesen. Die Israel-Mission dementierte die Behauptungen. 53

Nannens Darstellung überzeugt nicht. In einem israelischen Dokument vom 21. Februar 1965 wird darauf hingewiesen, daß die "Angelegenheit" (das Rüstungsgeschäft) 1962 zustande gekommen sei. Ben Gurion habe damals Peres zu Adenauer geschickt, der seinem israelischen Gast eine Zusicherung gegeben und Strauß schriftlich angewiesen habe, sein Möglichstes zur Umsetzung zu tun. Sämtliche Schritte seien Ausdruck "realistischer, sorgfältig abgewogener und vernünftiger deutscher Politik" gewesen. Selbstverständlich seien alle Aspekte der israelisch-deutschen Beziehungen behandelt worden, und die „Waffenlieferungen in beschränktem Umfang “, so Strauß angeblich, hätten dazu beigetragen, gefährliche psychologische oder politische Folgen des Eichmann-Prozesses für die Bundesrepublik zu verhindern. ${ }^{54}$ Während seines Besuchs in Jerusalem im Mai 1963 erwähnte Strauß in informellen Gesprächen eine Verständigung über Rüstungsgeschäfte. ${ }^{55}$ Bei einem Meinungsaustausch mit dem Auswärtigen Amt im Januar 1965 rechtfertigte Strauß die Rüstungsgeschäfte mit Israel einmal mehr mit dem Eichmann-Prozeß: „Die Israelis haben extreme Hetze gegen uns verhindert (Globke). ${ }^{\text {" } 56}$

Solange nicht das Gegenteil bewiesen werden kann, ist davon auszugehen, daß sich Jerusalem und Bonn irgendwie darauf verständigten, Globkes Verwicklung in den Eichmann-Prozeß zu verhindern. Loyalität war offenbar das Stichwort. Die israelische Seite hielt sich an die (ungeschriebene?) Abmachung, Globke aus dem Prozeß herauszuhalten, und wurde dafür von der deutschen Seite mit großzügigen Waffenlieferungen belohnt. Die israelische Strategie, sich auf Eichmann zu konzentrieren, andere, im öffentlichen Leben der Bundesrepublik stehende ehemals aktive Nationalsozialisten $z u$ verschonen und die von der Sowjetunion als revanchistisch und reaktionär bezeichnete Personalpolitik der Bundesrepublik zu ignorieren, wurde in Bonn zwar mit Wohlwollen registriert, fand aber im Kreml kein Verständnis. Die Sowjetunion erwartete die Verurteilung weiterer Nationalsozialisten - und erhoffte sich davon propagandistische Vorteile. Das Verhältnis zwischen den Sowjets und den „unkooperativen“ Israelis kühlte sich merklich ab. Der Kreml kritisierte das Verfahren und reagierte nicht auf die Gesuche der An-

53 Das israelische Außenministerium an die Außenstellen in Osteuropa vom 21.2. 1965; Fernschreiben der Mission in Köln an das israelische Außenministerium vom 21. 2. 1965; Tavor, Köln, an die Westeuropaabteilung vom 23. 2. 1965, ISA, 3533/4. Dieser Bericht enthält den Wortlaut von Nannens Erklärung und das Dementi der Israel-Mission.

54 Mission in Köln an das israelische Außenministerium vom 21. 2. 1965, ISA, 3533/4. Das Dokument wird auszugsweise in verschiedenen deutschen Zeitschriften so zitiert, daß es die Meinung des jeweiligen Autors wiedergibt.

55 PPP-Informationsbrief vom 19. 2. 1965, AdsD, SPD-Bundestagsfraktion, Akte Nr. 468; WELT AM SONNTAG (Hamburg) vom 21. 2. 1965; BONNER RUNDSCHAU vom 26. 2. 1965. Dr. Hans Stercken, Mitglied der Delegation am Eichmannprozeß und später Vorsitzender des Auswärtigen Ausschusses des Deutschen Bundestages, bestreitet in einem Schreiben an den Autor vom 14. 12. 1995, daß es eine solche Verständigung gab: „Es hat keine ,Verständigung' gegeben, da jedermann wußte, daß Globke nichts mit dem Fall Eichmann zu tun hatte. ${ }^{\circ}$

56 AAPD, Bd. 1, Dok. 2, S. 9. 
klage. ${ }^{57}$ Die anderen Ostblockstaaten zeigten sich zwar hilfsbereiter, insgesamt war der Sowjetblock der israelischen Prozeßführung und folglich auch Israel gegenüber jedoch feindlich gesinnt.

Der Fall Globke hielt die Bundesregierung in einem Zustand der Dauerspannung. Man hatte weder Gewißheit über die Pläne von Servatius noch über Eichmanns Absichten oder über das Wissen der Zeugen. Einiges Kopfzerbrechen bereitete Bonn Globkes angebliche Beteiligung an der Deportation griechischer Juden, und bei der Ankunft des ungarisch-jüdischen Historikers Jenoe Levai (in der Bundesrepublik oder in Israel?) trat der Bundesnachrichtendienst in Aktion, da angenommen wurde, daß Levai ein Manuskript über Globke mit sich führte, über dessen Inhalt aber niemand Näheres wußte. ${ }^{58}$ Die israelisch-deutsche Journalistin Inge Deutschkron bereitete dem Auswärtigen Amt viel Ungemach mit ihren unbequemen Fragen und Artikeln über Globke. Das größte Ärgernis war jedoch Kaul. Westdeutsche Journalisten versuchten, Kaul mit Gewalt zum Schweigen zu bringen. Seine Propaganda stand in direkter Konkurrenz zu westdeutschen Publikationen und Filmen. Von Preuschens Berichte offenbaren Besorgnis. Über jede Erwähnung Globkes im Prozeß wurde sofort nach Bonn berichtet. Der Umstand, daß die SS-Verantwortlichen für die Vernichtung des ungarischen Judentums unbehelligt in Freiheit lebten, und die Notwendigkeit ihrer Aussagen im EichmannProzeß, veranlaßten von Preuschen zu reger Diplomatie. Die ungeschickten Auftritte von Servatius brachten die deutschen Journalisten in Verlegenheit. Der einzige Lichtblick waren die Aussagen der deutschen "Gerechten unter den Völkern “ ${ }^{59}$ Von Preuschen tat sein Bestes auf diplomatischer Ebene. Die schrecklichen Enthüllungen über den Holocaust und die Furcht vor entsprechenden Reaktionen der Öffentlichkeit prägten die Stimmung.

\section{Die öffentliche Meinung}

Die Entführung Eichmanns und der nachfolgende Prozeß wurde von arabischer Seite mit Sorge aufgenommen, die das für arabische Belange besonders sensibilisierte Auswärtige Amt umgehend zu beschwichtigen versuchte. Die Kairoer Zeitung $E l$-Ahram übte als erste arabische Stimme scharfe Kritik an der Bundesregierung und beschuldigte sie, Eichmann billig an Israel verkauft zu haben. ${ }^{60}$ Die

57 Übersetzung des Zeitungsartikels „Prozeß oder Farce?" In: PRAWDA (Moskau) vom 28. 4. 1961, PA, 708, 82.70, 92.19; Kroll, Moskau, an das AA vom 28. 4. 1962; Übersetzung des Zeitungsartikels „Der Prozeß ist im Gange, aber...?“ In: KomsomolsKaJA PRAWDA vom 19. 5. 1961, PA, 708, 82.70, 92.19, Nr. 1038, Bd. 2; Die deutsche Botschaft in Moskau an das AA vom 28. 4. 1961, PA, L3, 80.00, 508/61, Bd. 3.

58 Levais Dokumente sollen auf dem Athener Flughafen unter mysteriösen Umständen verschwunden sein: Seelos, Athen, an das AA vom 4. 7. 1961, PA, L3, 80.00, 508/63, Bd. V, 30.6.61; L3, 80.00, 508/63, Bd. 5.

59 Bezeichnung des Jerusalemer Yad Vashem-Holocaust-Gedenkinstituts für Personen, die im Zweiten Weltkrieg Juden vor der Vernichtung retteten: die Botschaft in Mexiko an das AA vom 2. 4. 1961, PA, L3, 80.00, 508/63, Bd. 4.

60 EL-AHRAM (Kairo) vom 27. 5. 1960. 
Ägypter äußerten die Befürchtung, Israel könne den Prozeß gegen Eichmann dazu mißbrauchen, der Bundesrepublik „weitere Gelder abzupressen“. Zudem stellten sie die israelischen Zahlen über den Holocaust in Frage und kritisierten die Verbreitung "proisraelischer Propaganda“ durch die Bundesrepublik sowie Kauls Mission im Auftrag der DDR. Die Entführung Eichmanns bezeichneten die Ägypter als Akt der Piraterie und warfen der Bundesregierung vor, den Eichmannprozeß widerspruchslos hinzunehmen. Die libysche Reaktion offenbarte eine Mischung von Zorn und blankem Antijudaismus. Weitere zornige Reaktionen waren aus dem Irak, aus Saudi-Arabien und anderen arabische Staaten zu vernehmen. Einzig die libanesische Kritik klang relativ gemäßigt. Die Vertretungen der Bundesrepublik in arabischen Ländern berichteten über allgemeine Besorgnis und Unzufriedenheit angesichts des Eichmannprozesses in Israel und über wenig Verständnis für die gegen ihn erhobenen Vorwürfe.

Die Entführung Eichmanns und der Prozeß gegen ihn beschäftigte die Weltöffentlichkeit und führte in mehreren Ländern zu einer kritischen Auseinandersetzung mit der Vergangenheit. Das britische Außenministerium leitete z.B. eine Untersuchung über umstrittene Kapitel der eigenen Geschichte ein. Eine der untersuchten Fragen war, weshalb Großbritannien nicht auf ein Angebot Eichmanns eingegangen war, ungarische Juden im Austausch gegen vom NS-Regime dringend benötigte Güter ausreisen zu lassen. Die britische Regierung fürchtete, Israel könne den Alliierten vorwerfen, die Juden Hitler preisgegeben zu haben, und traf entsprechende Vorkehrungen. 61

Die vorhandenen Dokumente deuten darauf hin, daß die westdeutsche Gesellschaft den Eichmannprozeß als Last empfand. Der anfängliche Schock über die Entführung und die Eröffnungsphase des Prozesses wich später feindseligen Reaktionen, die man als Protest gegen das Aufrollen der Vergangenheit interpretieren könnte. Sehr verbreitet war eine Mischung aus Betroffenheit gepaart mit der Behauptung, nichts von den NS-Verbrechen gewußt zu haben. Um so tiefer saß dann der Schock angesichts der beim Prozeß zutage geförderten Greuel und führte diesmal zu Gesten der Reue, für die insbesondere die Kirchen verantwortlich waren, die ihre Gemeinden dazu aufriefen, der NS-Opfer zu gedenken und für sie zu beten. ${ }^{62}$ Eichmanns Hinrichtung wurde negativ aufgenommen, was besonders auch damit zusammenhing, daß die Todesstrafe in der Bundesrepublik abgeschafft war und ein deutscher Bürger gehängt wurde.

Die Auswirkungen des Eichmannprozesses auf die israelische Gesellschaft waren besonders einschneidend. Die nach dem Krieg verbreitete Verachtung der wehrlosen Massen der jüdischen Diaspora, die sich „wie Schafe zur Schlachtbank trieben ließen“, zusammen mit dem für die zionistische Hybris symptomatischen Ritual der Hochstilisierung des Widerstandes und der Verherrlichung des in Palä-

61 BRAHAM, Politics of Genocide; Memorandum „What to do in the case of appearance of the Joel Brandt story“ o.D., PRO, FO 371/151272, VR 1661/95; Ausarbeitung „The story of Alois Steiger" vom 20.12. 1960, PRO, FO 371/151272, VR 1661/97; die britische Botschaft in Den Haag an die Nahostabteilung vom 31. 12. 1960, PRO FO 371/159104.

62 Vogel an das Bundeskanzleramt und das Bundespresseamt vom 21./22. 6. 1961, PA, L3, $80.00,508 / 61$, Bd. 7 . 
stina geborenen "neuen jüdischen Menschen" fand ein jähes Ende, als der Eichmannprozeß die wahren Ausmaße der Katastrophe zutage förderte. Zum ersten Mal wurde der breiten Öffentlichkeit das Leid, die unbeschreibliche Angst und die Einsamkeit der Verfolgten konkret vor Augen geführt. Nach dem Eichmannprozeß etablierte sich der Holocaust als integraler Bestandteil des nationalen Ethos und des jüdischen Selbstverständnisses der Israelis. Der Prozeß hatte zudem auch langfristige Auswirkungen auf die Diaspora und führte das jüdische Schicksal einer breiten Öffentlichkeit vor Augen.

Schwer zu beurteilen sind die Auswirkungen des Eichmann-Prozesses auf die Einstellung der israelischen Gesellschaft gegenüber Deutschland. Manchmal gewinnt man den Eindruck, daß die beiderseitigen gesellschaftlichen Beziehungen nach dem Eichmann-Prozeß eine Wende zum Besseren genommen haben. Beobachter im Auswärtigen Amt meinten, die Israelis hätten gelernt, zwischen Nationalsozialisten und dem deutschen Volk zu unterscheiden. ${ }^{63}$ Diese Ansicht ist mit Vorsicht zu genießen. Wie sich zeigte, kamen die tiefsitzenden antideutschen $\mathrm{Ge}-$ fühle bei jeder ernsthaften Krise neu zum Vorschein. Der Eichmannprozeß führte den sonst fast ausschließlich mit dem Überleben des jungen Staates und dem persönlichen Wohlbefinden beschäftigten Israelis die tiefere Bedeutung ihrer jüngsten Vergangenheit und des gemeinsamen Erbes vor Augen. Insofern vertiefte der Eichmannprozeß den Graben zwischen den beiden Völkern. ${ }^{64}$

In den USA stationierte westdeutsche Diplomaten beurteilten die Reaktion der amerikanischen Öffentlichkeit und besonders auch des jüdisch-amerikanischen Publikums als weniger negativ wie ursprünglich befürchtet. Verschiedene innenund außenpolitische Entwicklungen verdrängten den Eichmannprozeß rasch aus den Schlagzeilen. Sehr unterschiedlich waren die Berichte aus Großbritannien, Skandinavien, Frankreich, Italien und Mexiko. Während die einen eine antideutsche Stimmung oder zumindest kühle Distanz zu Deutschland und gleichzeitig fehlendes Verständnis für Israel feststellen, lassen andere auf Unterstützung für Israel und eine kritische Haltung zur Bundesrepublik schließen. ${ }^{65}$ Aus einigen Ländern wurde kommentarlos berichtet. Die osteuropäische Presse hielt sich an die jeweilige Parteilinie und verwies auf die Genugtuung der jüdischen Seite über die strafrechtliche Verfolgung Eichmanns. Nur die ostdeutschen Zeitungen behandelten Eichmann, als wäre er Bürger irgendeines fernen Staates und völlig losgelöst vom deutschen Kontext. Gemäß der ostdeutschen Position gab es den Nationalsozialismus nur noch in der Bundesrepublik. Adolf Eichmann war demnach ein westdeutscher NS-Verbrecher. ${ }^{66}$

Adenauer verfolgte den Eichmannprozeß sehr aufmerksam. Er wandte sich zweimal direkt an die Öffentlichkeit, sprach einmal vor dem Bundestag und gab

${ }^{63}$ Gawlik an die deutschen Vertretungen im Ausland vom 30. 9. 1961, PA, L3, 80.00, 508/63, Bd. 4.

64 SHAHAM, Israel, S. 205.

65 HaARETZ (Tel Aviv) vom 30.7. 1961 und vom 15.-18. 12. 1961; Dawar (Tel Aviv) vom 15.-18. 12. 1961; von Etzdorf, London, an das AA vom 16. 11. 1961, PA, L3, 80.00, 508/ 63, Bd. 5; die Botschaft in Washington an das AA vom 15. 3. 1961, PA, L3, 80.00, 508/63, Bd. 5.

66 Timm, Hammer, S. 158-162. 
mehrere Erklärungen ab. ${ }^{67}$ Damit wollte der Kanzler die Öffentlichkeit überzeugen, daß Eichmann ein fairer Prozeß gemacht werde und daß der Staat Israel das Recht habe, den Mann vor Gericht zu stellen. Ein öffentlicher Appell Adenauers an Ben Gurion brachte aber auch Befürchtungen zum Ausdruck: Der Prozeß werde das „Urteil über uns Deutsche überhaupt" beeinflussen. ${ }^{68}$ Adenauer sorgte sich offensichtlich um den guten Ruf seines Volkes. Im Hinblick auf eine weitere Reise in die USA wies der Kanzler auf die Notwendigkeit gründlicher Öffentlichkeitsarbeit im eigenen Land hin. ${ }^{69}$ Auch Adenauer hätte am liebsten einen Schlußstrich unter die Kriegsverbrecherprozesse gezogen: „Dieser Eichmann-Prozeß hat uns ganz gewaltig geschadet, auch wenn es die Menschen nicht so hinausschreien. ${ }^{70}$ Für Ben Gurion lag die besondere Bedeutung des Prozesses an seinen erzieherischen Aspekten. Als überzeugter Zionist machte er das Exil bzw. die Diaspora für die Tragödie des jüdischen Volkes verantwortlich. ${ }^{71} \mathrm{Er}$ beschuldigte zudem die Nationalsozialisten, nicht die Deutschen, und wandte sich gegen Rassentheorien oder den Antisemitismus.

\section{Das Urteil}

Das Todesurteil gegen Eichmann wurde am 15. Dezember 1961 gefällt und am 31. Mai 1962 vom Obersten Gerichtshof bestätigt. Servatius reichte ein Gnadengesuch bei Staatspräsident Yitzchak Ben Zwi ein und löste damit eine neue Kontroverse aus. Eine kleine aber ausgesuchte Minderheit, die sich aus den angesehensten Vertretern der intellektuellen und akademischen Elite Israels zusammensetzte, unterstützte das Gesuch aus moralischen und emotionalen Gründen. In einem Brief an Ben Gurion betonten Martin Buber, Gerschom Scholem, Shmuel Hugo Bergmann und andere, daß Eichmann zwar keine Gnade verdiene, die Hinrichtung den Prozeß jedoch entwerte, seine historische und moralische Dimension verzerre und die Bedeutung des Holocaust dadurch zurückgestuft werde. Die Feinde des jüdischen Volkes seien an der Hinrichtung Eichmanns interessiert, da sie sich dann zur Behauptung berechtigt fühlten, die Sünden der Nationalsozialisten seien gesühnt und Blut mit Blut vergolten, schrieben die Gelehrten und fügten hinzu, daß der Vorstellung, wonach der Mord an sechs Millionen Menschen durch das Hängen eines einzigen Unmenschen gesühnt werden könne, kein Vorschub zu leisten sei. ${ }^{72}$

67 Meroz an Meir vom 14. 6. 1960, ISA, 4311/6; Hille an den Bundesminister vom 9. 3. 1961, PA, L3, 80.00, 508/61, Bd. 4; von Brentano an Adenauer vom 10. 3. 1961, BArch, N 1239, Bd. 158, Nr. 179; YEDIOTH ACHRONOTH (Tel Aviv) vom 14. 4. 1961.

68 DeuTSCHKRoN, Israel und die Deutschen, S. 123.

69 MENDE, Die neue Freiheit, S. 466-467.

70 AdENAUER, Teegespräche 1961-1963, S. 146; AAPD, Bd. 1, S. 595.

71 Israel Galili an Ben Gurion vom 25. 5. 1960, Ben Gurion an Israel Galili vom 26. 5. 1960, BGA, Correspondence File.

72 Martin Buber u.a. an Ben Gurion vom 26. 2. 1962, The National and University Library, Jerusalem, Martin Buber Collection, item no. 630. 
Die Mehrheit der israelischen Bevölkerung unterstützte dagegen die Hinrichtung mit Berufung auf historische Gerechtigkeit, Vergeltung und Rache, aber auch deshalb, um ein Zeichen für die Zukunft zu setzen. Der Leitspruch „Es gibt ein Gesetz und es gibt einen Richter" in Anlehnung an das Talmudzitat „es gibt weder Gesetz noch Richter" brachte die herrschende Stimmung treffend zum Ausdruck. Ein Volk, das über Jahrhunderte verfolgt wurde, nur weil es auf seine Art zu Gott beten wollte, an seinen eigenen Bräuchen festhielt und deshalb Neid und Fremdenfeindlichkeit hervorrief, sah die Stunde gekommen, endlich mit einem seiner Todfeinde abzurechnen. Eine Mischung aus Reue, Stolz, Kummer, Wut, Mitleid, Erinnerung und Erbarmen prägte das Verlangen des israelischen Normalbürgers, Eichmann am Strang zu sehen. Das mag nicht sehr edel gewesen sein, doch es zeugte vom langen Leidensweg des jüdischen Volkes. Der Staatspräsident lehnte das Gnadengesuch ab und antwortete mit folgendem Bibelzitat: „Wie dein Schwert Weiber kinderlos macht, so werde unter den Weibern auch deine Mutter kinderlos. ${ }^{" 73}$ Eichmann wurde am 31. Mai 1962 gehängt, die Leiche auf einem Torpedoboot der israelischen Armee auf See kremiert und die Asche außerhalb der israelischen Territorialgewässer ins Meer gestreut. Buber, der das Todesurteil nach dessen Vollstreckung weiter kritisierte, wurde Opfer einer Hetzkampagne der rechtsgerichteten Cherut-Partei. ${ }^{74}$

Der Eichmann-Prozeß war eine traumatische Erfahrung, sowohl für Israel als auch für die Bundesrepublik. Er vergegenwärtigte die Vergangenheit und konfrontierte die Menschen mit unbequemen Fragen. In Israel offenbarte er eine unbekannte Realität, und in beiden Ländern löste er alte Mythen durch neue ab. Der deutschen Bevölkerung diente der Eichmannprozeß als kollektiver Spiegel. Österreich, Hitlers Geburtsland und Heimat von Eichmann, Globocznik, Nowak und anderer SS-Führer, fühlte sich nicht angesprochen und nicht veranlaßt, irgendwelche historische Schlußfolgerungen zu ziehen.

Ein interessantes $Z$ wischenspiel in den deutsch-israelischen Beziehungen ist das Kapitel von Preuschen. Der Wiesbadener Rechtsanwalt reiste während des Eichmannprozesses regelmäßig nach Wiesbaden zu seinem privaten Anwaltsbüro und erstattete dabei auch seinem Freund von Brentano Bericht. Bei diesen Zusammenkünften entstand die Idee, die deutsche Beobachterdelegation in eine der IsraelMission in Köln entsprechende offizielle westdeutsche Dienststelle in Israel „übergleiten“ zu lassen. Von Preuschen stellte keine nennenswerten Einwände gegen diese Idee fest. ${ }^{75}$

Doch die israelische Seite war in Wirklichkeit weniger enthusiastisch. Nachdem diese Idee in den Jahren 1955/56 erwogen und schließlich verworfen wurde, war man nun nicht mehr bereit, sich mit weniger als mit einem Austausch von Bot-

73 Samuel, 1. Buch, 15, 33. Die Amalekiter galten als Erzfeinde der Israeliten auf ihrer Wanderung durch die Wüste. Die Nazis wurden von den Israelis mit den Amalekitern verglichen.

74 NEW YORK TIMES vom 5.6. 1962; CHERUT (Tel Aviv) vom 22.-27. 12. 1961 und 31. 5. 1962; HaAreTZ (Tel Aviv) vom 27. 12. 1961.

75 Varon an Shinnar vom 25. 5. 1961; Varon an Bendor vom 16. 6. 1961; der Leiter der Westeuropaabteilung an den Generaldirektor vom 18. 7. 1961, ISA, 3309/13. 
schaftern zufriedenzugeben. Shinnar war skeptisch. Gemäß seiner Einschätzung war das Auswärtige Amt aus Rücksicht auf die Araber und die Hallsteindoktrin weder bereit, von Preuschens Vorstoß zu akzeptieren noch ihn als Kandidaten für den entsprechenden Posten zu erwägen. ${ }^{76}$ Eine wenig überzeugende These vertritt der Historiker Rainer A. Blasius mit Berufung auf Dokumente aus dem Politischen Archiv des Auswärtigen Amts: Danach soll das israelische Außenministerium der Idee der Errichtung einer westdeutschen Dienststelle im Land positiv gegenübergestanden und der Beobachterdelegation Anfang Juni 1961 das Angebot gemacht haben, nach dem Eichmannprozeß in Israel zu bleiben. ${ }^{77}$ Dies sei vom Auswärtigen Amt und von Brentano übereinstimmend abgelehnt und als „Normalisierung über die Hintertreppe" bezeichnet worden sein. ${ }^{78}$ Das Auswärtige Amt soll erwogen haben, so Blasius weiter, nach Ablauf des Abkommens, irgendwo in Israel außerhalb von Jerusalem eine deutsche Handelsmission mit konsularischen Vollmachten zu errichten. Dem widerspricht die bereits im Zusammenhang mit der Politik Golda Meirs erwähnte israelische Maxime „Alles oder Nichts". Die Dokumente des israelischen Außenministeriums lassen jedenfalls nicht auf eine Änderung dieser Politik schließen.

Zweifellos erwog die israelische Regierung, von der mißlichen politischen Lage der Bundesregierung zu profitieren, wie dies auch bezüglich der Swastikawelle geschehen war. Yachil traf sich mit von Preuschen zum Gespräch über laufende bilaterale Fragen und versuchte die Delegation als Vehikel zur Übermittlung von Vorschlägen an das Auswärtige Amt zu benutzen. Doch das einzige greifbare Resultat der beiderseitigen Kontakte war offenbar die hinter dem Rücken des Auswärtigen Amtes erzielte Verständigung im Rüstungsbereich. Gemäß der Politik des Auswärtigen Amtes sollte die Lösung ausstehender Fragen während des Prozesses vermieden werden..$^{79}$ Alles in allem gewinnt man den Eindruck, daß das israelische Verfahren gegen Eichmann die Beziehungen zwischen Israel und der Bundesrepublik vertieft, die Verständigung gefestigt und das gegenseitige Vertrauen in heiklen Fragen gefördert hat. Zweifellos hat der Eichmannprozeß das Denken und Handeln beider Staaten geprägt.

76 Die Suche im PA nach Preuschens Vorschlag ergab keine Resultate. Dr. Stercken bemerkte dazu in einem Schreiben an den Autor vom 14. 12. 1995: „Bundesaußenminister von Brentano erwog die Idee, schließlich eine der israelischen Mission in Deutschland (sic!) entsprechende Vertretung in Israel zu errichten, aus anderen Gründen. Zudem handelte es sich weder um einen offiziellen Vorstoß, noch war die deutsche Delegation in Israel für diese Angelegenheit zuständig. "

77 BLASIUS, Geschäftsfreundschaft, S. 159.

78 Ebd.

79 Shinnar an Ben Gurion über den von Adenauer versprochenen Kredit vom 20. 5. 1961; Shinnar an Yachil vom 26. 5. 1961, ISA, 4316/5; Varon an die israelische Botschaft in Stockholm über den deutschen Botschafter Werkmeister und seine NS-Vergangenheit vom 16.7. 1961; Varon an Bendor betr. Werkmeister vom 16. 7. 1961, ISA 3309/11. 